Original Article

\title{
Evaluation of Electrolyte Imbalance in Acute Stroke
}

\author{
Mukhtiar Ahmed' ${ }^{1}$ Aziz-ur-Rehman², Safdar Ali Pervez ${ }^{1}$, Farrakh Imtiaz ${ }^{1}$, Abdul Razaque ${ }^{3}$ \\ Khawar Anwar ${ }^{4}$
}

${ }^{1}$ Department of Neurosurgery, Medicine and Community Medicine Khairpur Medical College, Pakistan. ${ }^{2}$ DOW Medical University, Karachi, Pakistan. ${ }^{3}$ Department of Neurosurgery Peoples Medical University of Health Sciences, Shaheed Banazirabad, Pakistan. ${ }^{4}$ Punjab Institute of Neurosciences (PINS), Lahore Pakistan

\begin{abstract}
Objective: Electrolyte imbalance is common in acute stroke; it may cause severe morbidity and mortality, so timely management of electrolyte imbalance may improve morbidity and mortality in cerebro-vascular accident. The purpose of this study was to evaluate electrolyte imbalance in acute stroke and its effect on the outcome of patients.
\end{abstract}

Material and Methods: This study was prospective descriptive in nature and it is comprised of 106 patients of acute stroke after clinically diagnosis confirmed by CT scan brain plain admitted in Khairpur Medical College Hospital. Serum electrolyte along with the other baseline investigation was performed.

Results: Hyponatremia was comparatively more common in brain infraction than spontaneous $\mathrm{ICH}$. Hypokalemia is also more common in brain infarction as compared to the hemorrhagic stroke patients of this study.

Conclusion: Incidence of Hyponatremia was more common than hypokalemia in patients with acute stroke.

Keywords: Hemorrhage, Infraction, Hyponatremia, Hypokalemia.

Corresponding Author: Mukhtiar Ahmed

Department of Neurosurgery, Khairpur Medical College

Email: ahmed.mukhtiar77@gmail.com

Date of Submission: 10-10-2020

Date of Revision: 25-11-2020

Date of Online Publishing: 25-12-2020

Date of Print: 31-12-2020

DOI: $10.36552 /$ pjns.v24i4.501

\section{INTRODUCTION}

A stroke is defined as an abrupt onset of neurologic deficit secondary to focal vascular cause there are two main types of acute stroke. First acute Infarction stroke caused by blockage of vessels and second hemorrhage stroke caused by rupture of blood vessels ${ }^{1}$. Stroke is a leading cause of disability and second most shared cause of death in developed and developing countries. ${ }^{2-}$

3 Almost 1 in 8 (11.9\%) deaths worldwide is caused by stroke. ${ }^{3}$

Early management of complications of strokemay improve outcome of patients, including brain edema, intracranial pressure, electrolyte imbalance, chest infection (aspiration 
pneumonia), malnutrition, bowel and bladder care, pulmonary embolism, deep vein thrombosis (DVT), pressure sores, joint stiffness and muscles contracture. $^{3}$

Disorder of sodium and potassium concentration is common electrolytes abnormalities in acute stroke. It can contribute to mortality if not corrected urgently. ${ }^{4}$ Electrolytes disturbances can occur due to conditions of elevated brain natriuretic peptide (BNP), syndrome of inappropriate anti-diuretic hormone. (SIADH) or cerebral salt wasting (CSW) syndrome, inappropriate fluid intake or loss leading to death and seizures. ${ }^{5-6}$ Intracerebral hemorrhage can associated with raised ICP and cause headache and vomiting further leading to dyselectrolytemia in acute phase of stroke. ${ }^{7}$ Development of Hyponatremia can causes further altered sensorium in stroke patients and when occur abruptly causes convulsions and aggravate cerebral edema leading to cerebral ischemia causing further brain damage and lead to death. ${ }^{8}$ Potassium is associated with inhibition of free radicals formation and modulates arterial vessel tone. ${ }^{9}$ Thus, acute stroke is a complex pathophysiological state and its management requires the efforts and skills of all the member of the

\begin{tabular}{|llccc|}
\hline \multicolumn{2}{|l}{ Table 1: Descriptive Results. } & & & \\
Variable & Options & Number & Percentage & Total \\
Gender & Male & 55 & 51.9 & $100 \%$ \\
& Female & 51 & 48.1 & \\
Age & $18-30$ years & 10 & 9.40 & \\
& $31-45$ years & 42 & 39.6 & $100 \%$ \\
Hemorrhage & Yes & 54 & 50.9 & \\
Infarction & Yes & 47 & 46.2 & \multirow{2}{*}{$100 \%$} \\
Serum Sodium & $111.5-135$ & 59 & 55.7 & \\
Level & $136-148$ & 47 & 55.7 & $100 \%$ \\
& $2.5-3$ & 6 & 44.3 & \\
Serum & $3-3.5$ & 18 & 5.7 & \\
Potassium & $3.5-4$ & 30 & 17.0 & \\
Level & $4-4.5$ & 30 & 28.3 & $100 \%$ \\
& $4.5-5$ & 22 & 28.3 & \\
\hline
\end{tabular}

multidisciplinary team. ${ }^{10}$ The purpose of this study was to evaluate electrolyte imbalance in acute strokeand its effect on the outcome of patients.

\section{MATERIAL AND METHODS}

\section{Study Design}

Prospective descriptive study. This study carried was out in Khairpur medical college hospital from January 2017 to December 2019. All patients admitted through the emergency department. Diagnosis of acute stroke confirmed by CT scan brain plain. Detail history and examination performed. Blood sample taken for the assessment of electrolyte and other baseline investigation send to rule out other associated diseases. Serum sodium between 136- 148 $\mathrm{mmol} / \mathrm{L}$ and potassium level between 3.5 $5 \mathrm{mmol} / \mathrm{L}$ considered as a normal range.

\section{Inclusion Criteria}

Patients above 17 years of age with acute stroke confirmed with $\mathrm{CT}$ scan brain were included in this study.

Patient with recurrent stroke transient Infarction attack and neurological deficit secondary to brain trauma were excluded from the study.

\section{Data Analysis}

SPSS system was used for data analysis.

\section{RESULTS}

In our study, 106 cases of acute stroke were selected after fulfillment of exclusion and inclusion criteria of study.

\section{Exclusion Criteria}




\section{Gender Distribution}

Table 1 showed that out of 106, male 55 (51.90\%) and female 51 (48.10\%).

\section{Age Incidence}

Ten patients were 18 to 30 years of age, 42 had age between 31 to 45 and 54 were $>45$ years.

\section{Clinical Presentation}

In this connection, the hemorrhage known cases were 47 and Infarction 59. Serum sodium level was between 111.5 to 135 in 59 patients and 136 to148 in 47 cases. Further, serum potassium level was between 2.5 to 3 in 6 cases, 3 to 3.5 in 18, 3.5 to 4 in 30,4 to 4.5 in 30 and 4.5 to 5 in 22 cases of acute stroke.

Table 2: Risk factors of acute stroke.

\section{Risk Factor}

Hypertension

Diabetes Mellitus

Dyslipidemia

Smoking

Table 2 revealed that out of 106 cases, 96 known cases of Hypertension out of 106, Diabetes Mellitus was seen in 45 patients, Dyslipidemia was observed in 57 patients and 43 known cases were smokers.

Table 3 showed the results of hemorrhage, Infarction, serum sodium level and serum potassium level in male and female patients with acute stroke.

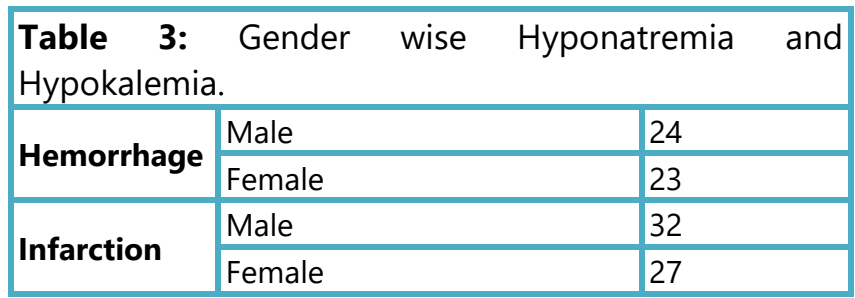

\begin{tabular}{|c|c|c|c|c|c|c|}
\hline \multirow{3}{*}{$\begin{array}{l}\text { Serum } \\
\text { Sodium }\end{array}$} & Level & \multicolumn{3}{|c|}{ 111.5-135 } & \multicolumn{2}{|c|}{$136-148$} \\
\hline & Male & \multicolumn{3}{|l|}{30} & \multicolumn{2}{|l|}{25} \\
\hline & Female & \multicolumn{3}{|l|}{29} & \multicolumn{2}{|l|}{22} \\
\hline \multirow{3}{*}{\begin{tabular}{|l} 
Serum \\
Potassium \\
Level
\end{tabular}} & Level & $2.5-3$ & 3-3.5 & 3.5-4 & $4-4.5$ & 4.5-5 \\
\hline & Male & 4 & 6 & 13 & 14 & 18 \\
\hline & Female & 2 & 12 & 17 & 16 & 4 \\
\hline
\end{tabular}

Below mentioned are the figures generated to represent data gender wise.

Table 4 is revealed the serum sodium level in Infarction patients and serum potassium level, it also showed the serum sodium level and serum potassium level in hemorrhage patients.

Table 4: Hyponatremia and Hypokalemia.

\begin{tabular}{|c|c|c|c|c|c|c|}
\hline \multirow{6}{*}{ 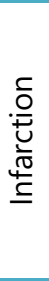 } & \multicolumn{4}{|c|}{ Serum Sodium Levels (mmol/L) } & \multirow{3}{*}{\multicolumn{2}{|c|}{$\begin{array}{l}\text { Total } \\
59\end{array}$}} \\
\hline & \multicolumn{2}{|c|}{ 111.5-135 } & \multicolumn{2}{|c|}{$136-148$} & & \\
\hline & \multicolumn{2}{|l|}{38} & \multicolumn{2}{|l|}{21} & & \\
\hline & \multicolumn{6}{|c|}{ Serum Potassium Level } \\
\hline & $2.5-3$ & $3-3.5$ & $3.5-4$ & $4-4.5$ & $4.5-5$ & 5 \\
\hline & 3 & 12 & 17 & 23 & 4 & 59 \\
\hline \multirow{6}{*}{ 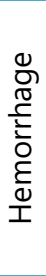 } & \multicolumn{6}{|c|}{ Serum Sodium Level } \\
\hline & \multicolumn{4}{|c|}{ 111.5-135 } & $136-148$ & \multirow{2}{*}{47} \\
\hline & 21 & & & & 26 & \\
\hline & \multicolumn{6}{|c|}{ Serum Potassium Levels (mmol/L) } \\
\hline & $2.5-3$ & $3-3.5$ & $3.5-4$ & $4-4.5$ & $4.5-5$ & 19 \\
\hline & 3 & 6 & 13 & 7 & 18 & 49 \\
\hline
\end{tabular}

\section{Risk Factors}

In this study, the age was most common risk factor, followed by hypertension in 96 patients out of 106.And diabetes mellitus in 45 patients out of 106 and dyslipidemia in 57 patients out of 106 and 43 patients were known cases of smoking.

Brain hemorrhages known cases were49 patients among them 24 were male and 23 were female, whereas, 57 patients were safe from it.

Brain ischemia was present in 59 cases that were 32 male and 27 female, on contrary 47 patients had not seen Infarction brain. As for the serum sodium level was concerned. 59 cases had 
serum sodium level between 111.5 to135 and among them 30 were male and 29 were female. Whereas, 47 patients had serum sodium level in between 136 to 148 . Whereas, 25 were male and 22 were female. Further, Serum Potassium Level was diagnosed, and its scale were 2.5-3, 3-3.5, 3.5-4, 4-4.5, 4.5-5 \& > $5 \mathrm{mmol} / \mathrm{L}$, respectively, in these known cases male were4, 6, 13, 14, 18, and female were $2,12,17,16,4$, respectively.

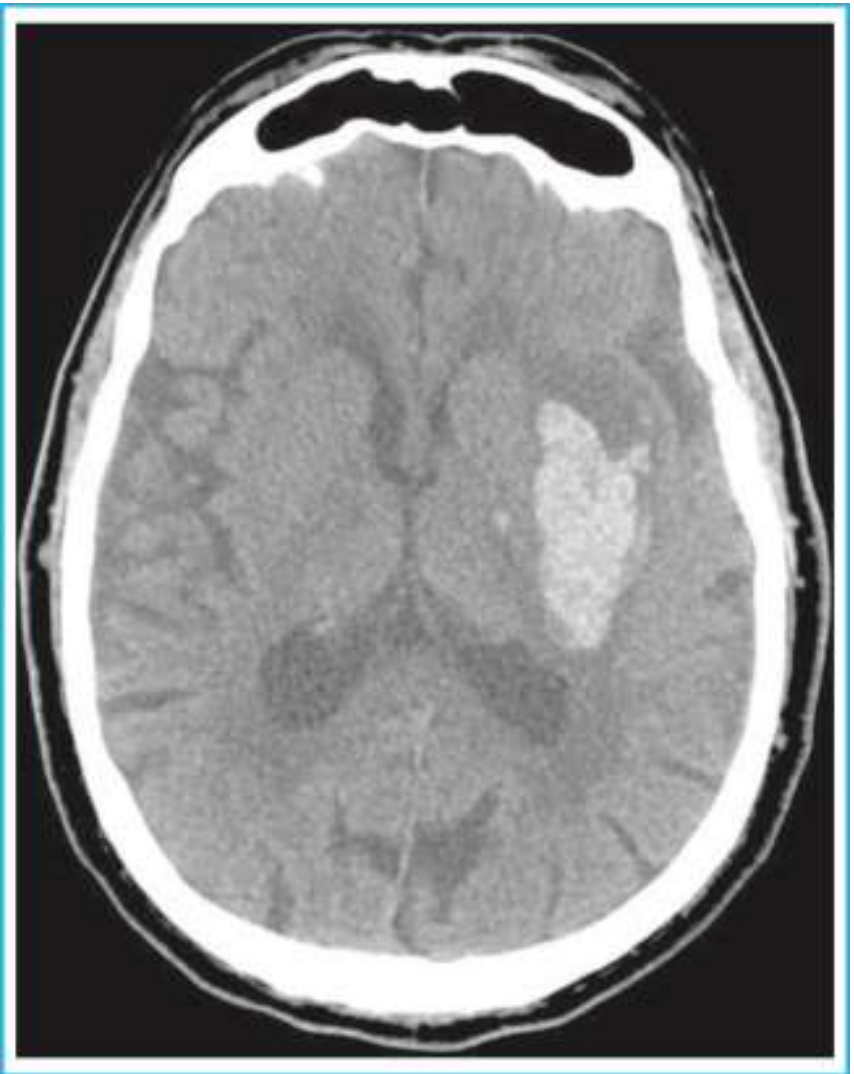

Figure 1: Left Intracerebral hemorrhage.

\section{DISCUSSION}

Acute stroke is acute onset of focal neurological findings in the vascular territory as a result of underlying cerebral vascular diseases. In this regard, Cerebro vascular accident is the third most common cause of death in developed countries after myocardial Infarction and cancer. ${ }^{12}$ This study was comprised of 106 patients with acute stroke. There are two main types, hemorrhagic and ischemic stroke. In this study, male patients were $51.9 \%$ and female $48.1 \%$. It was seen that men were more at risk than women for stroke. Similar outcomes were also seen in UK, USA, Italy, Saudi Arabia, Germany and Spain etc., ${ }^{18}$ but this difference was not at greater extend. Whereas, their ages were divided into three categories i.e. 18-30 (9.40\%), 31-45 (39.6\%) and $>45$ (50.9\%). This study resulted that vascular cerebral disease increases with increase in age. ${ }^{13}$ Unquestionably, this figure of acute stroke increasing worldwide, ${ }^{14}$ but, it is totally different from the result of Hassan et al. ${ }^{15}$ In this study, ischemic stroke (infarction) (55.7\%) cases were more than hemorrhagic stroke (46.2\%). Further, serum sodium and serum potassium level were checked of acute stroke patients. And then electrolyte imbalance was also seen gender wise. So in this study of acute stroke hyponatremia was seen in 30 male \& 29 female patients, but hypokalemia was seen in 10 male and 14 female patients with acute stroke. Same increasing rate of male patients was also other studies. ${ }^{18-20}$

In this study, acute stroke 47 patients were diagnosed as intra cerebral hemorrhage $(\mathrm{ICH})$ and 59 patients had brain infarction. Hypertension was the most common risk factor of acute stroke. $^{16}$ In our study, 96 (90\%) patients had hypertension out of 106 , but this rate of hypertension was $71.66 \%$ in another study. ${ }^{20}$ Diabetes Mellitus was seen in 45 patients, and Dyslipidemia was observed in 57 patients and 43 known cases of smoking.

In our study, 59 patients were of brain infarction where Hyponatremia was seen 38 patients having serum sodium level between 111.5 to $135 \mathrm{mmol} / \mathrm{L}$ and 21 patients were normal serum sodium level 136 - $148 \mathrm{mmol} / \mathrm{L}$. Hypokalemia was seen in 15 patients in which 3 patients had serum potassium level between range of 2.3 to 3 and in 12 patients to 3 to 3.5 $\mathrm{mmol} / \mathrm{L}$.

There were 47 patients of spontaneous $\mathrm{ICH}$ (hemorrhagic stroke) in which 21 patients of 
hyponatremia and their serum sodium level was 111.5 - $135 \mathrm{mmol} / \mathrm{L}$ and normal serum sodium level (136 mmol/L-148 mmol/L) in 26 patients.

Hypokalemia was seen in 9 patients, in which, 3 patients had serum potassium level between 2.3 to $3 \mathrm{mmol} / \mathrm{L}$ and 6 patients' serum potassium level 3 to $3.5 \mathrm{mmol} / \mathrm{L}$ out of 47 patients of $\mathrm{ICH}$. Patients with serum potassium levels below normal levels have poor outcomes. ${ }^{17}$ So, it is clear that the hemorrhagic group had a higher incidence of hyponatraemia. ${ }^{20}$

Results of this study are near to close with different scholars and researcher had seen hyponatremia was in $38.19 \%,{ }^{18}$ whereas, another study reported Hyponatremia in $11.60 \%,{ }^{19}$ but hypokalemia was observed in $15 \%$ patients.

In this study, the Hyponatremia was more common in ischemic stroke as well as in hemorrhagic stroke ${ }^{20}$ whereas, hypokalemia was less observed in both ischemic stroke and hemorrhagic stroke. ${ }^{20}$ In this way, in acute stroke patients the Hyponatremia was $38.19 \%$ and hypokalemia $15 \%$ and same is also relevant to other studies.

Early detection of electrolyte imbalance and careful monitoring is necessary to improve outcomes of acute stroke patients.

\section{Limitations}

Sample size of this study was small, and prolong follow up could be carried out. A definitive cause of electrolyte imbalance could not be sorted out.

\section{CONCLUSION}

Incidence of Hyponatremia was more common than hypokalemia in patients with acute stroke.

\section{REFERENCES}

1. Kalyan M, Nahdi WK, Kanitkar SA, Moharkar A, Saha R. Electrolyte Imbalance In Acute Stroke. National Journal of Integrated Research in
Medicine, 2017; 8 (4).

2. Smith W.S: Cerebrovascular diseases in: Harrison's Principle of Internal Medicine.19th Ed, Mc-Graw Hill Companies; New York City, 2015: 2559.

3. Monica W. Project Investigators. The world Health Organization MONICA Project (monitoring trends and determinant in cardiovascular diseases). J Clin Epidemiol. 2008; 41 (2): 105-14.

4. World Health Organization. The top 10 causes of deaths. 2014. Available at:

http://www.who.int/mediacentre/factsheets/fs310/ en/

5. Nemade ST, Patil MS, Chaudhari RA, Vankudre AJ. Serum electrolyte levels (sodium and potassium) in cerebrovascular accidents at a tertiary care hospital-a case control study. MVP Journal of Medical Science, 2016; 2 (1): 1-3.

6. Langhorne P, Stott DJ, Robertson et al. Medical complications after stroke: a multicenter study. Stroke, 2014; 31 (6): 1223-29.

7. WHO STEPS Stroke Manual: The WHO STEP wise approach to stroke surveillance. STEPS Stroke Surveil Man. 2016; 2 (1): 5-9.

8. Broderick J, Connolly S, Feldmann E, Hanley D, Kase C, Krieger D, Mayberg M, Morgenstern L, Ogilvy CS, Vespa P, Zuccarello M. REPRINT. Circulation, 2007; 116 (16): 391-413.

9. Stern RH. Severe symptomatic hyponatraemia. Treatment and outcome. A study of 64 cases. Ann Int Med. 2017; 107 (6): 656-64.

10. Coleman HA, Tare $M$, Tare $M$, Porkington HC. Endothelial potassium channels, endotheliumdependent hyperpolarisation and the regulation of vascular tone in health and disease. Clin Exp Pharmcol Physiol. 2014; 31 (9): 641e9.

11. Summers $D$, Leonard $A$, Wentworth $D$ et al. Comprehensive overview of Nursing and Interdisciplinary care of the acute Infarction stroke patients. A scientific statement from the American Heart Association. Stroke, 2009; 40 (8): 2911-44.

12. Hassan $M$, Hasan $A, \&$ Rubaiyat K. Electrolyte Disturbances in Acute Phase of Stroke Patients. Dinajpur Medical College Journal, 2013; 6: 12-16.

13. Huang $W$, Weng $W$, Peng $T$, Chien $Y$, Wu $C$, \& Lee M. Association of hyponatraemia in acute stroke stage with Three year mortality in patients with first ever Infarction stroke. Cerebrovascular Disease, 2012; 34: 55-60. 
14. Das BK, Sarder AH, Khanom T, Shaikh MS, et al. Variation of electrolyte status in relation to the type of acute stroke patients. Bangladesh Medical Journal Khulna, 2019; 52 (1-2): 12-15.

15. Siddiqui $M R$, Islam $Q T$, Haque $M A$, Iqbal $M J$, Hossain A, Rahman $Y U$, et al. Electrolytes status in different type of acute stroke patients and their correlation with some common clinical presentation. Journal of Medicine, 2012; 13 (2): 133-137.

16. Meenakshi K, Waleed K, Kanitkar S, Abhijit M, Rajdeb $\mathrm{S}$. Electrolyte imbalance in acute stroke. NJIRM. 2017; 8 (4): 23-6.

17. Longo-Mbenza B, Mbuilu Pukuta J, Lelo Tshinkwela M. Rates and predictors of strokeassociated case fatality in black Central African patients. Cardiovascular journal of Africa. 2008; 19 (2): 72.

18. Hassan AR, Aryan ZA. Electrolyte Disturbance in Hemorrhagic and Non-Hemorrhagic Stroke Patients in Al-Diwaniyah Teaching Hospital. Asian J Pharm Clin Res. 2018; 11 (1): 456-459.

19. Khan SN, Vohra EA. Risk factors for stroke: A hospital based study. Pak J Med Sci. 2007; 23 (1): 17-22.

20. Panda M, Sahu PK, Mandal MK, Mohapatra AK, Dany SS. Altered Serum Electrolyte Status in Acute Stroke Patients in Western Odisha, A Predictor of Syndrome of Inappropriate ADH (SIADH) or Cerebral Salt Wasting Syndrome (CSWS). Journal of Clinical \& Diagnostic Research, 2019; 13 (1).

\section{Additional Information}

Disclosures: Authors report no conflict of interest.

Ethical Review Board Approval: The study was conformed to the ethical review board requirements.

Human Subjects: Consent was obtained by all patients/participants in this study.

Conflicts of Interest:

In compliance with the ICMJE uniform disclosure form, all authors declare the following:

Financial Relationships: All authors have declared that they have no financial relationships at present or within the previous three years with any organizations that might have an interest in the submitted work.

Other Relationships: All authors have declared that there are no other relationships or activities that could appear to have influenced the submitted work.

\section{AUTHORSHIP AND CONTRIBUTION DECLARATION}

\begin{tabular}{|l|l|l|}
\hline Sr.\# & Authors Name & Intellectual/Contribution to Paper in Terms of: \\
\hline 1 & Mukhtiar Ahmed & Study design \\
\hline 2 & Aziz-ur-Rehman & Analysis of data. \\
\hline 3 & Farrakhlmtiaz & Literature review. \\
\hline 4 & Safdar Ali Pervez & Methodology. \\
\hline 5 & Abdul Razaque & Paper writing. \\
\hline 6 & Khawar Anwar & Literature review. \\
\hline
\end{tabular}

